\title{
DESENVOLVIMENTO DE INSTRUMENTO DE COLETA DE DADOS DE ENFERMAGEM PARA PACIENTES COM CÂNCER DE PULMÃO EM QUIMIOTERAPIA AMBULATORIAL
}

\author{
Developing a Nursing Data Colleting Instrument to Patients with \\ Lung Cancer Treated by Chemotherapy in Ambulatory \\ Desarrollo de Instrumento de Recogida de Datos de Enfermería \\ para Pacientes con Cáncer de Pulmón en Quimioterapia Ambulatoria
}

Anita Moda Salvadori ${ }^{1}$

José Luiz Tatagiba Lamas²

Cláudia Zanon ${ }^{3}$

\begin{abstract}
Resumo
0 câncer é um grave problema de saúde pública, e, dentre suas variedades, há o câncer de pulmão, que vem crescendo na população brasileira e mundial. A principal causa do câncer de pulmão é o tabagismo. Há três formas de tratamento: cirurgia, radioterapia e quimioterapia; para essa patologia a mais utilizada é a terceira. A enfermeira deve estar apta a lidar com as dificuldades desses pacientes, que durante o tratamento ambulatorial manifestam em seus domicílios efeitos colaterais. Os objetivos desta pesquisa são elaborar um instrumento de coleta de dados para pacientes com câncer de pulmão em quimioterapia ambulatorial e avaliar a validade de seus conteúdos e sua confiabilidade. Teve como guia a teoria do autocuidado de Dorothea Orem. Para sua validação, o instrumento foi primeiramente avaliado por juízes; após isso, foram realizados o pré-teste e o teste de confiabilidade pelo método da equivalência entre observadores. 0 instrumento se demonstrou confiável, considerando aquilo que se pretendia medir.
\end{abstract}

Palavras-chave: Processos de Enfermagem. Autocuidado. Quimioterapia. Neoplasias Pulmonares.

\begin{abstract}
Cancer is a serious public health problem and among its varieties there is lung cancer, which is increasing among brazilian people and in all the world. Its main cause is tobacco use. There are three ways to treat lung cancer: surgery, radiotherapy and chemotherapy. The last one is the most used. Nurses should be apt to deal with problems of the patients, who are exposed to collateral effects at home. demonstrating in their homes side effects. The objectives of this research are preparing an instrument for collecting data for patients with cancer of the lung in outpatient chemotherapy, and assess the validity of its content and its reliability. This work was guided by selfcare theory, by Dorothea Orem. The instrument was validated by five judges. After that, it was submitted to a previous test and its reliability was determined by equivalence between observers. The instrument demonstrated reliability, measuring the parameters it is supposed to.
\end{abstract}

Keywords:

Nursing Process. Self Care. Drug Therapy. Lung Neoplasms.

\section{Resumen}

El cáncer es un grave problema de salud pública y entre sus variedades existe el cáncer de pulmón, que está creciendo en la población brasileña y mundial. La principal causa de cáncer de pulmón es el tabaquismo. Existen tres formas de tratamiento, quirúrgica, por radioterapia y quimioterapia, siendo que para esta patología la más utilizada es la tercera. La enfermera debe estar habilitada para manejar dificultades de los usuarios que ante el tratamiento ambulatorio visiblemente pozo a su domicilio lado efectos. Los objetivos de esta investigación son elaborar un aparato desde colecta de datos para pacientes con cáncer de pulmón en quimioterapia en ambulatorio y evaluar la validad de sus contenidos y su confiabilidad. Tuvo como guía la teoría utilizada del auto-cuidado de Dorothea Orem.Para que sea comprobada la validez de dicho instrumento primeramente este fue evaluado por jueces, luego fue realizado un pre-test y el test de confiabilidad por el metodo de equivalencia entre los observadores. El instrumento demostró realmente su confiabilidad midiendo aquello a lo que se lo propuso a medir.

Palabras clave: Procesos de Enfermería. Autocuidado. Quimioterapia. Neoplasias Pulmonares.

'Enfermeira, Universidade Estadual de Campinas, Mestre em Enfermagem pela Faculdade de Ciências Médicas FCM - UNICAMP- Campinas SP. ${ }^{2}$ Enfermeiro, Prof. Doutor, Departamento de Enfermagem, Faculdade de Ciências Médicas, Universidade Estadual de Campinas, SP. ${ }^{3}$ Enfermeira, Ambulatório de Quimioterapia, Hospital das Clínicas, Universidade Estadual de Campinas, SP. 


\section{INTRODUÇÃO}

0 câncer é uma doença que vem sendo muito discutida e um grave problema de saúde pública, sendo a segunda causa de morte por doença no Brasil. Embora as maiores taxas de incidência de câncer sejam encontradas em países desenvolvidos, cerca de $55 \%$ dos casos novos são encontrados em países em desenvolvimento. Encontramos na literatura tumores que são mais comuns em países em desenvolvimento (câncer de colo de útero, pênis, estômago e cavidade oral) e outros mais incidentes em países desenvolvidos (câncer de mama, pulmão, próstata e cólon e reto). No Brasil, encontramos tanto tumores típicos de países desenvolvidos como em desenvolvimento ${ }^{1,2}$.

0 câncer de pulmão é comum entre os tumores malignos, apresentando um aumento por ano de $2 \%$ na sua incidência mundial. No Brasil, o câncer de pulmão, traquéia e brônquios teve uma estimativa de incidência para o ano de 2006 de 23.150 casos novos entre homens e de 12.300 entre as mulheres, sendo que as maiores taxas são encontradas no Mato Grosso do Sul, São Paulo, Rio de Janeiro, Espírito Santo, Santa Catarina, Paraná e Rio Grande do Sul2. É importante salientar um crescimento superior a $100 \%$ de mortos por essa doença no sexo feminino ${ }^{2-5}$. Em 2004, o câncer de pulmão foi a décima causa de morte entre os homens no país e a décima primeira entre as mulheres ${ }^{2}$.

A maioria dos pacientes, na ocasião do diagnóstico, apresentase com a doença em estágio avançado, o que dificulta o tratamento. Estudos nacionais e internacionais têm mostrado uma sobrevida que, em geral, não ultrapassa cinco anos ${ }^{6-8}$.

Existem três formas de tratamento: cirurgia, radioterapia e quimioterapia. Esta última modalidade, vinculada a esta pesquisa, tem se tornado uma das mais importantes e promissoras maneiras de combater o câncer. É um tipo de tratamento sistêmico, diferentemente da cirurgia e da radioterapia, que são mais antigas e de atuação localizada.

Os fármacos mais utilizados para tratamento do câncer de pulmão apresentam principalmente neurotoxicidade, depressão de medula óssea (leucopenia, anemia, trombocitopenia, granulocitopenia), ototoxicidade, nefrotoxicidade, distúrbios eletrolíticos, efeito emetogênico, estomatite, diarréia, alopécia, febre e flebite.

A quimioterapia é vista pelos pacientes como um importante fator estressante, no entanto também é vista como um tratamento necessário. É importante que a pessoa seja capaz de realizar um bom enfrentamento no primeiro contato com a quimioterapia. Caso contrário, pode haver piora nos efeitos colaterais das aplicações seguintes ${ }^{9,10}$.

Uma assistência de enfermagem com acurada identificação de problemas relacionados ao estado físico, espiritual, mental e psicossocial dos pacientes pode oferecer esperança para alívio dos sintomas através de um programa de intervenção bem estruturado. Para isso é necessário que a assistência de enfermagem se guie por uma teoria que possa englobar essa demanda, como a proposta por Dorothea Orem sobre o autocuidado.

Dorothea Orem foi a responsável pelo desenvolvimento da teoria do autocuidado. Ela referiu que a enfermagem tem uma especial preocupação com o autocuidado dos pacientes. Sua teoria engloba 0 autocuidado, a atividade de autocuidado e a exigência de autocuidado, bem como requisitos para o mesmo, e ela está dividida em três partes relacionadas: autocuidado, deficiências do autocuidado e sistemas de enfermagem.

Na primeira parte, ela definiu o que é o autocuidado e suas vantagens, apresentando três categorias de requisitos de autocuidado: universais, desenvolvimentais e de desvio de saúde. Os requisitos universais estão relacionados com necessidades básicas do ser humano, como respiração e ingestão suficiente de água e alimento, dentre outros. Os requisitos desenvolvimentais são aqueles agregados a processos naturais, como o envelhecimento. 0 autocuidado por desvio de saúde é aquele que surge em condições de doença, ferimento ou moléstia, que podem ser permanentes ou transitórios. Um exemplo de desvio de saúde transitório é um paciente internado por apendicite: ele tem um desvio de saúde que, após a recuperação do procedimento cirúrgico, lhe permite seguir uma rotina igual à anterior. 0 segundo tipo de desvio de saúde (permanente) está relacionado com doenças crônicas (hipertensão, diabete) ou acometimento permanente devido a algum trauma (paraplegia, amputação). 0 câncer é definido como um desvio de saúde permanente porque é uma doença crônica que, mesmo controlada, precisará de acompanhamento médico por toda a vida, pois uma pessoa que já apresentou um tumor maligno tem um risco aumentado de desenvolver outro ${ }^{9,10}$. Encontramos diversos trabalhos na literatura que mostram 0 uso da teoria de Orem em pacientes com doenças crônicas ${ }^{11-13}$.

Há seis requisitos para o autocuidado por desvio de saúde: busca e garantia de assistência adequada; conscientização e atenção aos efeitos e resultados de condições e estados patológicos; execução efetiva de medidas prescritas; conscientização e atenção, ou regulagem de efeitos desagradáveis ou maléficos de medidas prescritas; modificação do autoconceito (e da auto-imagem), na aceitação de si como estando num estado especial de saúde e necessitando de formas específicas de cuidado de saúde; aprendizado da vida associado aos efeitos de condições e estados patológicos, bem como de efeitos de medidas de diagnóstico e tratamento, num estilo de vida que promova o desenvolvimento contínuo do indivíduo $0^{9,10}$.

Na segunda parte, Orem descreveu a necessidade de intervenção de enfermagem quando o paciente apresenta um déficit de autocuidado, ou seja, está incapacitado ou limitado para realizar seu autocuidado. Neste contexto, Orem identificou cinco métodos de ajuda: agir ou fazer para o outro; guiar o outro; apoiar o outro (física ou psicologicamente); proporcionar um ambiente que promova 0 desenvolvimento pessoal, quanto a se tornar capaz de satisfazer demandas futuras ou atuais de ação; ensinar o outro ${ }^{14}$.

Na terceira parte de seu trabalho, Orem apresentou a teoria dos sistemas de enfermagem, na qual os pacientes são incluídos, pela enfermeira, de acordo com suas necessidades de autocuidado, nos sistemas totalmente compensatório, parcialmente compensatório e de apoio-educação. Os três sistemas podem ser aplicados a um mesmo indivíduo. 0 paciente pode passar por todos os sistemas num curto período de tempo, não concomitantemente, de acordo com o desenvolvimento de seu estado ${ }^{10}$.

Em seu trabalho, Orem também discutiu os conceitos de ser humano, saúde, sociedade e enfermagem e definiu três 
passos para o processo de enfermagem: diagnóstico e prescrição; planejamento de um sistema de enfermagem e para execução de cuidados; e produção e gerenciamento dos sistemas de enfermagem ${ }^{10}$.

Os pacientes que são encaminhados para a quimioterapia antineoplásica chegam com grandes demandas emocionais e físicas, dúvidas quanto ao tratamento e suas conseqüências, expectativa de melhora, incerteza quanto ao futuro e, ainda, sofrem os efeitos colaterais dos fármacos muitas vezes em casa. Notamos que eles têm um contato breve com o enfermeiro por estar em um ambiente ambulatorial. Com isso, muitos apresentam déficit de conhecimento, o que os leva a apresentarem problemas físicos e emocionais. Isto se faz presente tanto no paciente como no cuidador, que não receberam orientações adequadas sobre como agir diante de diversos problemas que o tratamento traz. Logo, necessitam de uma assistência de enfermagem efetiva e estruturada, para que os sintomas sejam minimizados ou enfrentados com eficiência. A teoria do autocuidado, elaborada por Orem, parece ser um caminho para alcançar uma assistência de enfermagem mais eficaz, mas há necessidade de um instrumento de coleta de dados que torne todo o processo viável. Logo, este estudo tem como objetivos: elaborar um instrumento de coleta de dados para pacientes com câncer de pulmão em quimioterapia ambulatorial e avaliar a validade de conteúdo do instrumento e sua confiabilidade do instrumento.

\section{METODOLOGIA}

A pesquisa foi realizada no ambulatório de quimioterapia do Hospital das Clínicas da Universidade Estadual de Campinas (UNICAMP), após aprovação do projeto pelo Comitê de Ética em Pesquisa da Faculdade de Ciências Médicas da Universidade Estadual de Campinas. A população foi composta por adultos de ambos os sexos, portadores de câncer de pulmão. Para compor a amostra, vinte sujeitos foram escolhidos aleatoriamente no período de 05/06/2006 a 10/07/2006. Foram critérios de inclusão ter o câncer de pulmão como diagnóstico primário, ter participado de ao menos uma seção de quimioterapia, ter acima de 18 anos e aceitar participar da pesquisa, assinando o termo de consentimento livre e esclarecido.

Inicialmente foi elaborado um instrumento de coleta de dados, baseado em uma revisão da literatura sobre o assunto, sendo que os principais estudos utilizados foram os de Bajay ${ }^{15}$, Hermida $^{14}$ e Silva ${ }^{16}$. Também foram levadas em consideração as características clínicas e as necessidades dos pacientes com câncer de pulmão, e a teoria de Dorothea Orem, que serve como guia para a coleta de dados. 0 instrumento é composto de dados gerais sobre o tratamento (que contemplam requisitos de autocuidado por desvio de saúde), requisitos de autocuidado universais (que incluem dados de anamnese, segurança emocional e higiene pessoal) e exame físico (que contempla requisitos de autocuidado desenvolvimental e por desvio de saúde). Foi elaborado também um guia que contém questões para orientar o raciocínio sobre os dados coletados, visando identificar problemas, formular diagnósticos de enfermagem e elaborar prescrição de enfermagem. No entanto, consideramos que o enfermeiro deve ser capacitado para desenvolver seu próprio raciocínio clínico, a fim de que o instrumento possa ser utilizado com maior efetividade.

0 instrumento contém dados gerais (situação conjugal e escolaridade), aspectos de autocuidado nos desvios de saúde (diagnóstico médico, tipo de quimioterapia, quimioterápico utilizado, via de administração, intervalo entre as aplicações e fase do tratamento em que o paciente se encontra) e requisitos de autocuidado universais (padrão de sono e repouso, exercícios físicos, hábitos, padrões nutricionais/hidratação, atividade diária, relacionamento familiar, espiritualidade, segurança emocional e higiene pessoal). Também consta 0 exame físico, composto de sinais vitais, exame da cabeça/pescoço/ neurológico, nutrição/hidratação, tórax/pulmões, abdome, eliminações, extremidades/dorso e localização de feridas.

$\mathrm{Na}$ segunda etapa foi verificada a validade de conteúdo. Os instrumentos foram submetidos à apreciação de cinco juízes, todos com título de doutor, e os contatos foram realizados por meio de correio eletrônico. Os juízes registraram suas opiniões e sugeriram a retirada, acréscimo ou modificação dos itens. Os dados obtidos foram submetidos à análise estatística por meio do coeficiente de Kappa e testes de Friedman e de Kendall. Foram adotadas modificações sugeridas nas quais os testes estatísticos forneceram resultados significativos na avaliação de concordância entre os juízes.

O pré-teste foi realizado no ambulatório de quimioterapia do Hospital das Clínicas da UNICAMP, e a amostra foi composta de 10 pacientes.

Para essa fase do estudo e a coleta de dados final, o instrumento foi dividido em duas partes. A primeira, contendo dados gerais e requisitos de autocuidado universal, foi preenchida apenas pelo pesquisador, já que as respostas eram dadas pelo paciente e qualquer pessoa que estivesse ouvindo anotaria o mesmo resultado.

$\mathrm{Na}$ etapa seguinte foi avaliada a confiabilidade da segunda parte do instrumento, correspondente ao exame físico. A confiabilidade foi testada por meio do método da equivalência, que consiste na aplicação simultânea do instrumento por dois observadores diferentes ${ }^{16}$. 0 instrumento demonstra equivalência quando a concordância entre os observadores é alta. Os dados foram coletados pela pesquisadora e por uma enfermeira do ambulatório de quimioterapia, com grande experiência na área. Esse mesmo sistema de validade de conteúdo e avaliação da confiabilidade do instrumento de coleta de dados já foi utilizado por outros autores ${ }^{15-17}$.

A análise estatística destes dados foi realizada por meio do coeficiente Kappa e teste de Wilcoxon.

\section{RESULTADOS E DISCUSSÃO}

Os dados sócio-demográficos confirmam o que encontramos na literatura: o câncer de pulmão é mais comum entre homens com idade avançada, já que a média de idade foi de 61,4 anos ${ }^{1,6}$, e em pessoas com baixo nível de escolaridade. Inquérito domiciliar realizado entre os anos de 2002 e 2003 mostrou que o hábito de fumar é mais freqüente entre pessoas com menos de oito anos de escolaridade, ou seja, aquelas que não completaram o ensino fundamental ${ }^{15}$. Além disso, nossos dados 
foram coletados em uma instituição que atende pacientes de toda região pelo Sistema Único de Saúde (SUS), gratuitamente. Em geral, pessoas atendidas por instituições como essa apresentam baixo nível sócio-econômico e baixo grau de escolaridade.

A maior parte da população do estudo referiu problemas para manter o sono, muitas vezes relacionado com dispnéia durante a noite devida à patologia de base. No entanto, afirmaram que dormem em quantidade suficiente. Segundo Levin, pacientes com câncer de células não pequenas avançado apresentam problemas de sono com maior freqüência, da quarta à sétima sessão de quimioterapia ${ }^{19}$.

Diversos pacientes, quando interrogados sobre a razão de não praticarem atividade física, referiram dispnéia e principalmente cansaço nas pernas. É bastante lógico que a dispnéia seja um dos principais sintomas apresentados por portadores de câncer de pulmão. Além disso, a fadiga é apontada como um dos efeitos colaterais dos quimioterápicos ${ }^{23,24}$.

Alterações relacionadas com a emotividade foram pouco encontradas, provavelmente porque o pouco tempo de contato entre entrevistador e entrevistado não permitiu a formação de um vínculo. 0 paciente necessita de ajuda em muitas funções que antes realizava sozinho, mas com os efeitos dos medicamentos e a progressão da doença já não consegue mais, necessitando da ajuda de um cuidador que acaba restringindo com o paciente sua qualidade de vida ${ }^{25}$.

Sem dúvida, a principal causa do câncer de pulmão é o tabagismo, o que também ficou claro nesta pesquisa, já que $95 \%$ da amostra referiu ser ou ter sido tabagista'. Também podemos observar a forte relação que existe entre o consumo de cigarro e de café, já que a maior parte da população estudada fazia uso dos dois.

0 consumo de líquido foi insuficiente para a maior parte da população, o que é um fato preocupante e ao mesmo tempo esperado. Poucas pessoas têm o hábito de consumir dois litros ou mais de líquido por dia. Muitos quimioterápicos são nefrotóxicos, dentre eles a cisplatina, que foi uma das drogas utilizadas pelos pacientes deste estudo. A baixa ingesta de líquido pode aumentar a toxicidade desta droga. 0 restante das drogas utilizadas tem uma maior relação com a depressão da medula óssea ${ }^{24}$.

A gestação deve ser evitada durante o tratamento quimioterápico, pois as drogas são teratogênicas e mutagênicas, e a criança pode nascer com sérios problemas físicos ou mentais dependendo da idade gestacional em que se inicia o tratamento. Na vigência de gravidez, aumenta o risco de morte do paciente e aumenta a chance de progressão da doença, podendo levar à morte. Por outro lado, mesmo que a gravidez fosse levada até o final, a mulher poderia não ter condições físicas e emocionais para cuidar de seu filho ${ }^{6}$. Atualmente alguns estudos demonstram que o tratamento não altera o curso biológico da doença, ou seja, não aumenta e nem diminui a sobrevida materna e não afeta o feto tão drasticamente como se imaginava, sendo seus efeitos mais danosos quando aplicado no primeiro trimestre de gestação. Entretanto, o câncer é, sabidamente, uma doença que espolia as reservas nutricionais do paciente, reservas que já estariam sofrendo forte demanda adicional em função da gravidez. Em nosso estudo havia duas mulheres que tinham vida sexual ativa e nenhuma delas fazia uso de algum método contraceptivo, pois já haviam passado pela menopausa.

De um modo geral, a concordância entre os observadores $A$ e $B$ com relação à pressão arterial foi satisfatória, pois pequenas diferenças entre medidas são aceitáveis. Entretanto, alguns itens apresentaram concordância baixa ou intermediária, o que justifica uma análise mais aprofundada.

\section{Tabela 1:}

Valores de média, desvio-padrão (dp), mínimo, mediana e máximo de pressão arterial sistólica e das diferenças (Dif) entre observadores A e B. $n=20$. Campinas, 2006.

\begin{tabular}{cccccc}
\hline Variável & média & dp & mínimo & mediana & máximo \\
\hline PAS-A & 126,3 & 22,8 & 100 & 119 & 190 \\
PAS-B & 123,5 & 23,2 & 90 & 120 & 190 \\
Dif & 2,8 & 7,9 & -10 & 0 & 24 \\
\hline
\end{tabular}

$p=0,1709$ (Wilcoxon para amostras relacionadas)

\section{Tabela 2:}

Valores de média e desvio-padrão (dp), mínimo, mediana e máximo de pressão arterial diastólica e das diferenças (Dif) entre os observadores A e B. $n=20$. Campinas, 2006.

\begin{tabular}{lllccc}
\hline Variável & média & dp & mínimo & mediana & máximo \\
\hline PAD - A & 79,5 & 16,3 & 60 & 74 & 120 \\
PAD - B & 78,8 & 14,9 & 60 & 70 & 110 \\
Dif & 0,8 & 4,1 & -10 & 2 & 10 \\
\hline
\end{tabular}

$p=0,3526$ (Wilcoxon para amostras relacionadas)

Em ambas as Tabelas 1 e 2 encontramos uma concordância satisfatória.

A concordância foi baixa com relação ao pulso e temperatura. Os dois tipos de medidas foram realizados pelos dois observadores simultaneamente. No caso da temperatura, os dois observadores olharam o mesmo termômetro seqüencialmente. Acreditamos que a baixa concordância pode estar relacionada a arredondamentos ocorridos, que tenham alterado sutilmente as médias calculadas. Como a temperatura interna é regulada pelo organismo dentro de limites muito estreitos, um décimo de grau pode de fato significar uma diferença clínica ${ }^{26}$. Entretanto, isso é questionável, considerando que a temperatura foi medida na região axilar, local em que a variação aceita como limite de normalidade é superior a um grau ${ }^{27}$.

Quanto ao pulso, cada pesquisador palpou uma artéria radial, simultaneamente. Houve baixa concordância, sendo que o observador A obteve uma média de 83,2 bpm (dp 13,9), enquanto o observador B contou 91,5 bpm (dp 16,3). Como não são esperadas, em condições normais, diferenças significativas neste parâmetro entre os dois braços, a baixa concordância pode estar relacionada a algum problema cardiovascular do paciente ou a alguma conseqüência de sua doença. Houve um pesquisado que apresentou pulso muito arrítmico, o que pode ter feito com que os pesquisadores apontassem valores distintos para o mesmo paciente. 
Por fim, salientamos que não se pode descartar o fator humano como um dos motivos que produziram a baixa concordância entre esses itens.

Apesar de ocorrerem algumas respostas distintas, a concordância foi considerada ótima quando se tratou de ruídos pulmonares, edema de membros inferiores, dentição incompleta e ausência de dentes. Nesses dois últimos itens, a concordância não foi total, provavelmente por algum observador ter considerado ausência de dentes como dentição incompleta ou não ter observado com atenção a cavidade bucal do paciente.

Visão turva, higiene bucal, coloração de pele, perfusão periférica e locomoção tiveram uma concordância intermediária porque se referem a dados que necessitam de uma avaliação subjetiva dos observadores. 0 mesmo ocorre com a hidratação do paciente, que apresentou uma fraca concordância. Hermida ${ }^{14}$, em sua dissertação de mestrado, também realizou a validação de um instrumento que continha o exame físico e, em relação à higiene bucal, também encontrou baixa concordância ${ }^{14}$.

A ausculta pulmonar apresentou algumas diferenças, mas os dados não puderam ser analisados devido ao grande número de variáveis e à baixa freqüência de casos em cada item. Entretanto, as discordâncias encontradas foram poucas e podem ter sido devidas à interpretação dada ao som auscultado.

Quanto ao padrão intestinal, as poucas divergências foram relacionadas com certa indefinição do paciente. Quando se perguntava, por exemplo, "com que freqüência vai ao banheiro evacuar?" ele respondia "uma ... duas a três vezes por dia".

\section{Referências}

1. Guerra MR, Gallo VM, Azevedo G, Mendonça L. Risco de câncer no Brasil: tendências e estudos epidemiológicos mais recentes. Rev Bras Cancerol 2005 jun/jul/ago; 51(3): 227 -34.

2 Ministério da Saúde (BR) Instituto Nacional do Câncer. Estimativa 2006: incidência de câncer no Brasil. [on-line] [citado 20 nov 2006]. Disponível em: http://www.inca.gov.br.

3. Ministério da Saúde(BR) Instituto Nacional do Câncer. Atlas de mortalidade por câncer no Brasil:1979- 1999. Rio de Janeiro (RJ): INCA; 2002.

4. Ministério da Saúde (BR). Instituto Nacional do Câncer. Estimativa da incidência e mortalidade por câncer no Brasil. Rio de Janeiro (R); INCA; 2003.

5. Ministério da Saúde(BR) Instituto Nacional do Câncer. Apresenta informações sobre câncer. [on- line] [citado 17 maio 2005]. Disponível em http://www.inca.gov.br.

6. Ayoub AC, Fontes ALC, Silva MAA, Alves NRC, GigliotteP, Silva YB. Planejando o cuidar na enfermagem oncológica. São Paulo (SP): Lemar; 2000.

7. Curbelo ONM, Vidal RA, Ronquillo HAC. Sobrevida del carcinoma de pulmón no células pequeñas sometido a cirurgía en relacion con el estadio clínico. Rev Cubana Oncol 2001 abr; 17(1): 39-42.

8. Sadler AB. Chemotherapy for small cell lung cancer. Sem Oncol 2003 Feb; 30(1): 9-25.

9. Orem DE. Nursing: concepts of pratice. $4^{\mathrm{a}}$ ed. New York (USA): Mosby Year Book; 1985.

10 Foster PC, Janssens, NP. Dorothea E. Orem. In: George JB. Teorias de enfermagem: os fundamentos para a prática profissional. Porto Alegre (RS): Artes Médicas; 1993.
Isso levou um observador marcar 2 a 3 vezes por dia como resposta, enquanto o outro anotava 1 a 3 vezes por dia. No trabalho de Hermida (2005), foi possível avaliar este item, sendo obtida uma boa concordância ${ }^{14}$.

A maior parte dos itens do instrumento, no que diz respeito ao exame físico, apresentou concordância total. Os trabalhos de Bajay (2002) e Hermida (2005) não discriminam exatamente em quantos pontos dos instrumentos desenvolvidos foi encontrada concordância de $100 \%$, mas apontam a ocorrência muito freqüente de valores de Kappa acima de 0,75, como aconteceu também nesta pesquisa ${ }^{14,15}$.

\section{CONCLUSÕES}

0 referencial de Dorothea Orem mostrou-se eficaz na elaboração de um instrumento de coleta de dados para pacientes com câncer de pulmão em quimioterapia ambulatorial.

Embora na primeira parte do instrumento não tenha sido feito o teste de confiabilidade, ele se mostrou eficaz para conhecer o paciente, o tipo de tratamento e os requisitos de autocuidado universais.

Na segunda parte do instrumento, embora tenha havido alguns pontos de fraca concordância, o teste de confiabilidade revelou concordância boa, ótima ou total entre os observadores.

Enfim, o instrumento elaborado nesta dissertação pode servir como guia na coleta de dados de pacientes com câncer de pulmão em quimioterapia ambulatorial e auxiliar na Sistematização da Assistência de Enfermagem desses pacientes.

11. Coelho MS, Silva DMGV. Grupo educação-apoio: visualizando o autocuidado com os pés de pessoas com diabetes mellitus. Cien Cuid Saude 2006 jan; 5(1): 11-15.

12 Figueiredo AE, Kroth LV, Lopes MH. Diálise peritoneal: educação do paciente baseada na teoria do autocuidado. Science Medical $2005 \mathrm{jul} /$ ago/set; 15 (3): 198-202.

13 Freire MRSM, Nóbrega MML. 0 idoso hipertenso e o autocuidado Rev RENE: revista da rede de enfermagem do nordeste $2001 \mathrm{jan} / \mathrm{jul} ; 2$ (1): 60-68.

14. Hermida PMV. Elaboração e validação de instrumento para coleta de dados na assistência de Enfermagem. [dissertação de mestrado]. Campinas (SP): Universidade Estadual de Campinas/ UNICAMP; 2005.

15. Bajay HM, Rosa KM, Araújo IEM, Lamas JLT, Queiroz MCC, Bianchin MRA. Elaboração e implementação de roteiro de exame físico de enfermagem. Programas e Resumos do Encontro de Enfermeiros de Hospitais de Ensino do Estado de São Paulo. Campinas (SP); 2002. p. 21.

16. Silva SR. Assistência de enfermagem a acompanhamento domiciliar a pacientes de câncer ginecológico, submetidos à quimioterapia antineoplásica. [tese de doutorado] Ribeirão Preto (SP): Escola de Enfermagem/USP; 2000.

17. Radovanovic CAT. Desenvolvimento e validação de um instrumento para avaliar a movimentação e transferência de pacientes: uma abordagem ergonômica. [dissertação de mestrado]. Campinas (SP): Universidade Estadual de Campinas/ UNICAMP; 2002.

18. Cherneck C. Temporal differences in coping, mood, and stress with chemotherapy. Cancer Nurs 1999 Aug; 22(04): 266-76.

19. Fried TR, Bradley EH, Towle VR. Valuing the outcomes of treatment. Arch Intern Med 2003 Sept; 163 (22): 2073-78. 
20. Lobiondo-Wood G, Haber J. Pesquisa em enfermagem: métodos, avaliação crítica e utilização. $4^{a}$ ed. Rio de Janeiro (R)): Guanabara Koogan; 2001.

21. Ministério da Saúde(BR). Instituto Nacional de Câncer. Coordenação de Prevenção e Vigilância. Inquérito domiciliar sobre comportamentos de risco e morbidade referida de doenças e agravos não transmissíveis: Brasil, 15 capitais e Distrito Federal- 2002-2003. Rio de Janeiro (RJ): INCA; 2004.

22 Levin RD, Daehler MA, Grutsch JF, Quiton CG, Lis CG, Peterson DG, et al. Circadian function in patients with advanced non-small-cell lung cancer. Br J Cancer 2005 Jun; 93(11): 1202-208.

23. Bonassa EMA. Enfermagem em terapêutica oncológica, $2^{\mathrm{a}}$ ed. São Paulo (SP): Atheneu; 2000.
24. Fonseca SM, Machado RCL, Paiva DRS, Almeida EPM, Massunaga VM, Koike CT, et al. Manual de quimioterapia antineoplásica, enfermagem prática. Rio de Janeiro (RJ): Reichmann \& Affonso Ed; 2000.

25. Zang AY, Siminoff LA. The role of the family in treatment decision making by patients with cancer. Oncol Nurs Forum 2003 Feb; 30(6):1022-028.

26. Guyton AC. Fisiologia humana e mecanismos das doenças. Rio de Janeiro (RJ): Guanabara Koogan; 1998.

27. Porto CC. Exame clínico: bases para a prática médica, $4^{\mathrm{a}}$ ed. Rio de Janeiro (RJ): Guanabara Koogan; 2000. 